\title{
Femme de tête et homme de cœur
}

\author{
Agata Jackiewicz \\ Université Paris-Sorbonne \\ STIH \\ Agata.Jackiewicz@paris-sorbonne.fr
}

\section{Introduction}

Parmi les manifestations de la subjectivité langagière, les plus évidentes et les plus clairement avouées sont celles qui sous-tendent des jugements d'opinion ou de valeur. Cette problématique, malgré les apports significatifs dûs notamment aux travaux de Catherine Kerbrat-Orecchioni (Kerbrat-Orecchioni 2002) et de Patrick Charaudeau (Charaudeau 1992, 2011), reste encore relativement peu explorée en linguistique française. Depuis quelques années cependant, les recherches portant sur les discours évaluatifs se trouvent fortement motivées par les multiples enjeux sociétaux de la veille d'opinion et de l'E-réputation. On peut consulter à ce propos un récent numéro thématique de la revue TAL $(53: 1,2010)$, intitulé « Opinions, sentiments et jugements d'évaluation ».

L'étude que nous présentons ici se situe à la frontière du traitement automatique des langues et de la linguistique et prend place dans le cadre du projet ANR OntOpiTex ${ }^{1}$. Ce projet vise l'extraction automatique et la caractérisation sémantique fine des segments textuels porteurs d'opinions et de jugements d'évaluation. Une série d'analyses et d'expérimentations informatiques associées portant sur les constituants détachés de nature axiologique qui ciblent des individus ou des organisations ont été menées dans ce cadre (Jackiewicz et al. 2009), (Ferrari et al. 2009), (Jackiewicz 2010). Ces travaux ont fait émerger des structures récurrentes au moyen desquelles il est possible d'identifier l'expression des qualités associées à des personnes. Il s'agit en quelque sorte de témoins linguistiques des valeurs que la société considère comme désirables ou indésirables au cours d'une époque. Analyser le fonctionnement de ces structures, grâce à des explorations systématiques sur des corpus étendus, permet de construire une certaine image de ces valeurs.

Les jugements d'évaluation dont font l'objet les individus concernent leur dynamique propre de sujets singuliers, mais se font également à la lumière de leur appartenance socio-professionnelle. Comme être social, l'individu répond aux projets, aux normes, aux règles (loyautés invisibles ${ }^{2}$ de I. BoszormenyiNagy ; habitus $^{3}$ de P. Bourdieu) que son milieu (familial, social, professionnel...) d'appartenance lui commande de respecter. L'identité sociale comprend les attributs catégoriels ou statutaires (sexe, âge, métier...) qui se réfèrent à des catégories sociales où se rangent les individus ("jeunes », "étudiants », « femmes », « cadres », « pères », « catholiques »...). Il n'est donc pas rare de rencontrer des discours évaluatifs qui tentent de faire correspondre informations catégorielle et individuelle.

Des expressions telles que homme de concept, patron à poigne, brillant économiste, en politicien expérimenté... peuvent apparaître en apposition, dans le rôle d'une prédication seconde (Neveu 1998), complétant (étayant, justifiant...) ce qui est dit à propos de l'entité dénotée par le sujet de la phrase principale.

Dans cette optique, nous allons nous intéresser à une construction spécifique "homme| femme de $\mathrm{N}(+\mathrm{exp})^{4} »$, qui associe à un terme catégoriel ou typant (homme ou femme) un terme qualifiant (courage, culture, convictions...). Les expressions construites sur ce patron sont rencontrées, tant dans la presse que dans la littérature, dans divers types d'écrits et notamment ceux qui relèvent des " récits de vie », (biographie, portrait, nécrologie, en vue...), voir (1).

(1) H.B.-M. a été l'un des grands patrons de la presse de ce siècle. Homme d'exception, il symbolisait à lui seul l'idéal d'indépendance et de probité. Homme de courage, il avait su 
résister aux pressions des pouvoirs politiques et des groupes financiers pour maintenir haute et toujours vivante l'exigence de liberté.

De par sa structure, cette construction peut être questionnée de deux points de vue. Tout d'abord, et c'est notre interrogation principale, quelles propriétés/qualités « attache »-t-on, à ce niveau de catégorisation, à des individus et quelles fonctions remplissent-elles (classifiante, axiologique...) ? Ensuite, observe-t-on dans les discours évaluatifs une dissymétrie de fonctionnement selon la catégorie (ici, le genre) de la cible ? Autrement dit, existe-t-il des qualités qui sont prioritairement attachées à l'une ou à l'autre de ces catégories ? Retrouve-t-on (dans les corpus étudiés) des traces des représentations stéréotypées (au sens de Amossy et Herschberg 2011) qui sont durablement associées à des individus de chaque sexe ? Il est évident que les informations ainsi obtenues sont valables relativement au cadre temporel et sociétal « couvert» par le corpus choisi. Par ailleurs et plus généralement, quelles sont les caractéristiques essentielles des énoncés qui comportent ce type d'expression?

Pour répondre à ces questions, nous avons collecté automatiquement sur corpus des attestations de cette structure, afin d'en faire une première analyse quantitative et qualitative. L'approche choisie est entièrement guidée par les données. Nous avons fait le choix d'analyser la construction « (hommeffemme) de $\mathrm{N}(+\exp )^{5} »$, sans déterminant et avec ou sans expansion, quand elle est placée en apposition. Conforme à ces critères, elle est attestée dans le corpus (20 ans du Monde) 2887 fois $^{6}$. L'extraction a été réalisée par Thierry Charnois et Stéphane Ferrari du laboratoire GREYC, avec l'outil LinguaStream.

Cette démarche est à la fois différente et complémentaire des approches qui choisissent pour observables des données lexicographiques (TLFi, EuroWordNet, par exemple), comme c'est le cas chez (Baider et Jacquey 2008). Elle permet de travailler sur des usages langagiers effectifs et actuels, observés dans des contextes déterminés (socio-culturels, politiques, professionnels...).

Précisons que le travail décrit ici ne constitue pas un but, mais un moyen (c.à.d. une étude de faisabilité) pour pouvoir définir ultérieurement des stratégies informatiques efficaces permettant d'explorer et d'annoter automatiquement dans des corpus textuels de grand volume des expressions potentiellement porteuses de contenus évaluatifs. Dans cette optique, nous cherchons à identifier les formes pertinentes et celles qu'il faudra écarter des traitements (par exemple, les entités polylexicales comme femme de ménage ou homme d'affaires). Il est utile d'avoir un aperçu de la variabilité lexicale relative à la construction étudiée au sein du corpus choisi. De même sur le plan sémantique, on interroge à cette étape des travaux la pertinence et la manière d'aborder la question de la polarité (positive, négative ou neutre) des expressions qualifiantes. Le classement a priori et hors contexte de ces expressions est-il possible ? Sera-t-il efficient et utile?

\section{Principales propriétés et variantes formelles}

En première approximation, la structure «(hommelfemme) de $\mathrm{N}$ », compacte et synthétique, tend à sélectionner les qualités (dénotées par $\mathrm{N}$ ou par l'expression complète) à un certain niveau de généricité? Comme l'illustrent les expressions réunies dans le paradigme (a), cette caractéristique la distinguerait des tours analogues, tels que par exemple « réputé/connu... pour GN/GV », qui n'exercent pas de contrainte quant au degré de spécification de la qualité attribuée.

(a)
Homme de courage,
Homme de génie,
Homme de dossiers,
Admiré pour son courage,
Connu pour être un génie ou presque,
Homme de gauche,
Reconnu pour sa connaissance des dossiers,
Homme de poigne,
?Homme de poigne de fer,
?Homme de rigueur idéologique communiste,
Connu pour son militantisme de gauche,
Connu pour sa poigne,
Connu pour sa poigne de fer,
Connu pour sa rigueur idéologique communiste,

On peut remarquer aussi que cette forme de qualification est peu compatible avec des restrictions temporelles (homme de courage *en 2009/*depuis hier) et semble relativement étanche à des 
modalisations (*probablement homme de courage). Ces caractéristiques vont dans le sens d'une spécialisation permettant d'exprimer des qualités essentielles ou durablement associées à des personnes, au sein des jugements établis ou relativement consensuels. A l'évidence, l'information véhiculée par de tels jugements touche à la réputation ${ }^{8}$ des individus concernés.

Rappelons que ce rapport particulier au temps se manifestant par une incompatibilité avec des prédicats indiquant l'extension temporelle caractérise la sémantique des noms de qualité.

\begin{abstract}
«Ce que la langue traduit par ce blocage, c'est une conséquence de la liaison essentielle de chaque chose avec ses qualités : si les qualités n'ont pas elles-mêmes de durée, c'est que ce sont les choses, avec les qualités, qui durent ou ne durent pas. Les qualités d'une personne ou d'une chose ne changent pas, c'est la personne ou la chose elle-même qui change. » (Flaux et Van de Velde, 2000, 81).
\end{abstract}

La construction «(hommelfemme) de $\mathrm{N}(+\exp )$ »employée en apposition peut présenter des variantes. L'extraction automatique a été configurée de manière à pouvoir identifier toutes les expansions possibles pouvant être introduites par la préposition de. Après un premier examen des données collectées, nous pouvons distinguer trois familles de configurations.

Il y a, tout d'abord, la construction minimale qui associe au mot tête un terme simple (b) ; son expansion sera notée « de $\mathrm{N}$ ». Elle est présente dans 1178 énoncés (soit $41 \%$ des observables).

(b) de courage, de parole, de tête, de poigne, de main, de rêve, de caractère, de pouvoir, d'influence, de révolte, de paille, de passion, de culture, d'action, de paradoxes...

Dans 1122 énoncés (39\%), le terme N s'accompagne d'une forme expansée, susceptible de plusieurs fonctions (modifiant le terme tête, le terme $\mathrm{N}$, l'expression complète...). La plus fréquente est la construction «de $\mathrm{N} \operatorname{Adj}$ », viennent ensuite : de $\mathrm{N}$ qui..., de $\operatorname{Adj} \mathrm{N}$, de $\mathrm{N} \operatorname{Adv}$, de $\mathrm{N} \operatorname{Adv} \operatorname{Adj}$, de $\mathrm{N}$ à..., de $\mathrm{N}$ sans..., de tous les $\mathrm{N}$, de $\mathrm{N}$ ayant..., de $\mathrm{N}$ s'il est est/fut, de $\mathrm{N}$ par..., de $\mathrm{N}$ Adj et Adj, de $\mathrm{N}$ que... de N Vpartprés... (c).

(c) de petite vertu, d'action efficace, d'action d'abord, d'affaires très fortuné, d'ouverture s'il en est, de dialogue par tempérament, de tous les mauvais coups, de dialogue discret et compétent, de droite sans états d'âme...

Ce catalogue des formes n'est pas exhaustif étant donnée la possibilité d'imbrications (de toutes les métamorphoses que l'on n'arrivera jamais à enfermer dans un rôle). On note que les expansions en qui, que, sans, à/au/aux, ayant introduisent souvent d'autres qualités (d), mais c'est le cas aussi de certaines formes avec une expansion adjectivale (homme d'action efficace).

(d) d'exception à l'esprit incandescent, de caractère qui ne cachait pas son athéisme, de gauche sans avoir jamais adhéré au PS...

Dans 587 énoncés (20\%), l'expansion contient plusieurs substantifs. Il s'agit principalement de deux configurations «de $\mathrm{N} 1$ et de $\mathrm{N} 1$ » et « de N1 et N2», présentes dans 534 énoncés. Dans les 9\% d'énoncés restants, ces deux qualités sont articulées entre elles sur d'autres modes que la conjonction simple. Nous en avons identifiées plusieurs (plus que de, autant que de, autant que, ou de, ou, plus que, et non de, mais, plutôt que de, mais aussi de, mais aussi, avant d'être, bien plus que, devenu, puis, tout autant que...) (e).

(e) de silence et de solitude, de cour et humaniste, d'exception ou banale entremetteuse, d'amour mais aussi de violence, de pudeur mais aussi homme de devoir, de consensus plus que d'affrontement, de convictions plutôt qu'idéologue, de culture autant qu'homme de pouvoir, d'édition tout autant qu'écrivain, de cinéma devenu homme de théâtre...

Ce classement a pour objet (i) d'apprécier la complexité formelle des expressions (pour la construction des grammaires plus fines), (ii) d'identifier les configurations les plus fréquentes, (iii) d'attirer l'attention sur celles où l'interprétation de la polarité passe nécessairement par un calcul complexe. 


\section{Termes qualifiants : quels champs sémantiques ? Quelle charge axiologique?}

A travers l'étude de la construction «homme|femme de $\mathrm{N}$ », nous cherchons principalement à identifier les qualités potentiellement axiologiques qui sont véhiculées par ce support. Ces qualités, considérées comme saillantes ou tenues pour valorisantes ou dévalorisantes, sont actualisées dans les textes choisis pour l'étude, lesquels reflètent ou véhiculent, partiellement, certains systèmes de valeurs en cours dans la société. Nous tenterons, malgré les limites évidentes liées à l'objet étudié et son support d'expression, d'identifier les champs sémantiques que recouvrent les qualités attestées. Il s'agit d'une caractérisation relativement générale donnant accès à des domaines qualitatifs, plutôt que d'une description fine de l'ensemble du lexique collecté (ce qui présuppose des moyens humains plus importants).

Il s'agit également d'apprécier la charge axiologique des expressions bâties sur cette construction. Or, cette tâche s'avère particulièrement délicate, car elle est difficilement envisageable sans tenir compte de l'énoncé d'accueil et du contexte extralinguistique. Certaines qualités peuvent être (i) valorisées ou non, (ii) valorisées positivement ou négativement, selon le contexte (social, professionnel, culturel) où est produit l'acte évaluatif. Le cas des expressions qui renvoient à des modes de fonctionnements socioprofessionnels (homme de pouvoir, homme de réseaux, homme de terrain, homme de dossiers...) est à ce titre particulièrement éclairant.

Dans (2), homme de terrain est essentiellement distinctif. Le point de vue émanant d'un homme de terrain est différent de celui qui vient d'un homme de sciences. Dans (3), la teneur positive de cette expression vient de l'opposition avec technocrate, terme chargé négativement. Dans (4) enfin, elle participe à une composition relativement positive à laquelle contribuent également les qualificatifs syndicalisme de proximité et attentif aux pratiques professionnelles.

(2) L'un, homme de terrain, affirma que le volcan de Guadeloupe grondait, certes, mais qu'il n'était pas nécessaire d'évacuer les populations. L'autre, homme de sciences, conclut que, si le pire n'était pas sûr, il n'en convenait pas moins, selon le principe de précautions, d'évacuer.

(3) Ce militaire de carrière, homme de terrain, n'a rien d'un technocrate. Son franc-parler en fait un patron du renseignement hors du commun.

(4) Issu d'une grande "fédé », homme de terrain, attaché au syndicalisme de proximité et attentif aux pratiques professionnelles, F.C. devrait être davantage porté sur les questions d'organisation.

Le cas des qualités à axiologie fluctuante (instable ou sensible au contexte) devra donc être considéré au même titre que ceux des qualités clairement orientées sur l'axe des polarités.

Plus généralement, sans pouvoir leur accorder toute la place nécessaire, nous tenterons de montrer l'influence de certains paramètres co-textuels qui jouent dans l'interprétation de nos observables.

\section{Catégories sémantiques}

Les données attestées ont été traitées en plusieurs étapes, incluant lecture, codage, tri et interprétation. Ce travail a conduit à une première catégorisation sémantique, de nature clairement tendancielle (tableau 1).

\begin{tabular}{|l|r|r|}
\hline Catégorie & Occ & \multicolumn{1}{c|}{$\%$} \\
\hline Autre & 135 & $5 \%$ \\
\hline Ax & 1078 & $37 \%$ \\
\hline Ob & 846 & $29 \%$ \\
\hline QI & 828 & $29 \%$ \\
\hline Total & 2887 & $100 \%$ \\
\hline
\end{tabular}

Tableau 1: Les fréquences des attestations selon le type sémantique de l'expression 
La catégorie axiologique $(\mathrm{Ax})$ réunit des constituants qui dénotent des qualités dotées d'une charge positive ou négative (homme de confiance, femme de courage...). Ces qualités n'ont pas le pouvoir de fonder des classes d'individus. L'ensemble (Q1) correspond aux expressions qualifiantes sans charge axiologique a priori (homme de lettres, femme de gauche, homme de presse...), ayant un certain pouvoir classifiant. Ces expressions renvoient à l'appartenance experte à des champs d'activités ou de compétences et à des univers idéologiques. Enfin, la catégorie $(\mathrm{Ob})$ réunit des formes qui dénotent des caractéristiques objectives et discriminantes. Il s'agit d'un nombre très restreint d'expressions lexicalisées qui renvoient à des métiers et des fonctions, ainsi qu'au statut de "compagne de " (femme de ménage, homme d'affaires, femme de gendarme, femme de prisonnier...). Dans la catégorie (Autre), nous avons classé les expressions composites, à cheval entre les classes $(\mathrm{Ql})$ et $(\mathrm{Ob})$, telles que homme de confiance de $\mathrm{Y}$ ou homme de main de $\mathrm{Y}$.

Il est important de préciser qu'en contexte approprié, les expressions de type (Q1) peuvent recevoir une interprétation axiologique. Dans (5), il y a mention de deux cadres d'interprétation différents et concurrents, chacun possédant son propre système de valeurs. De même, il est évident que l'image (positive ou négative) associée à certains métiers $(\mathrm{Ob})$ peut contribuer à une interprétation axiologique des formes qui y font référence (homme d'affaires, femme de ménage).

(5) Cet homme respecté à Wall Street (il est l'ancien gouverneur du New York Stock Exchange) a toujours été considéré par les républicains comme un dangereux "libéral " (homme de gauche) partisan du rôle actif de l'Etat dans l'économie, bref, un défenseur de ce "Big Government " devenu la bête noire du Grand Old Party.

On retiendra à ce stade de l'étude que deux tiers des attestations de la construction « homme|femme de $\mathrm{N}(+\exp ) »$ sont susceptibles d'une interprétation axiologique.

Cette typologie, croisée avec les deux termes catégoriels, donne pour les attestations collectées la répartition explicitée dans le tableau 2.

\begin{tabular}{|l|c|c|c|c|c|c|}
\hline \multicolumn{1}{|c|}{ Tête } & $\mathrm{Ob}$ & Autre & $\mathrm{Ql}$ & $\mathrm{Ax}$ & \multicolumn{1}{c|}{ Total } & \multicolumn{1}{c|}{$\%$} \\
\hline femme & $234(28 \%)$ & $2(1 \%)$ & $50(6 \%)$ & $145(13 \%)$ & 431 & $15 \%$ \\
\hline homme & $612(72 \%)$ & $133(99 \%)$ & $778(94 \%)$ & $933(87 \%)$ & 2456 & $85 \%$ \\
\hline Total & 846 & 135 & 828 & 1078 & 2887 & $100 \%$ \\
\hline
\end{tabular}

Tableau 2 : Les fréquences des attestations réparties selon le type sémantique de l'expression et suivant le terme catégoriel

La construction «(homme|femme) de $\mathrm{N}$ » véhicule donc majoritairement des jugements ciblant des individus de genre masculin ${ }^{9}(85 \%$ contre $15 \%)$. Il est à noter que cette proportion générale est comparable à celle qui caractérise les attestations de nature axiologique (87\% vs 13\%). Dans les emplois qualifiants, l'écart entre les deux genres se creuse davantage ( $94 \%$ contre $6 \%)$.

\subsection{Emplois axiologiques $(\mathrm{Ax})$}

\subsubsection{Polarité}

Certaines occurrences de la structure « (hommeffemme) de $\mathrm{N} »$ véhiculent assez nettement un jugement de valeur. Toutefois, cette évidence tient souvent à une lecture globale de l'énoncé, plus qu'à un calcul compositionnel fait sur certains ou sur l'ensemble de ses membres (6).

(6) Le président de la République a rendu hommage à cette "personnalité exceptionnelle, homme de cour, de conviction et de foi qui a su mettre ses actes en conformité avec ses engagements et soulever des montagnes. 
L'interprétation de la polarité des énoncés axiologiques avec «homme de $\mathrm{N}$ » ou «femme de $\mathrm{N}$ » dépend a minima de la signification du terme $(\mathrm{N})$ et de l'influence du cotexte. Comme nous venons de le voir, la teneur axiologique de $\mathrm{N}$ est fonction de plusieurs paramètres. Quant au cotexte, son orientation peut être, par exemple, « superficiellement » positive, mais susceptible de plusieurs lectures en fonction du contexte extralinguistique (7), plus clairement négative, mais présentant des limites de validité (temporelles, par exemple) (8), contrastée (9-10) avec ou sans polarité dominante.

(7) Homme de réseaux, pur produit de l'élite française, il semble (...)

(8) Homme de clan, de réseau, H.K. a longtemps souffert d'une sorte de gaucherie, d'un manque d'à-propos, raillés pendant des années dans les milieux intellectuels et politiques de tout bord.

(9) Homme de morale, en effet, sachant fustiger et revenir sur ses méchancetés, reconnaître ses torts, éviter les raideurs du gauchisme en vogue au plus fort de son activité, et ne pas tenir rigueur aux confrères qui s'y complaisaient...

(10) Mais J.-Y.H., homme de pouvoir, brillant directeur du Trésor, président de Paribas, a-t-il été cinq années durant une potiche à la tête du Lyonnais, sans prise sur la réalité ? Son ambition qui n'était pas mince, son souci de la grandeur de la banque, ont amplifié les travers d'un établissement incapable de mesurer les risques pris.

Le classement des énoncés polarisés, malgré les limites d'une telle opération (liées à la subjectivité de l'analyste), s'avère globalement justifié, car il fait apparaître des tendances marquantes. Le tableau 3 en montre une qui tient en l'occurrence à la très faible part des segments de polarité négative.

\begin{tabular}{|l|r|r|r|r|r|}
\hline Tête & \multicolumn{1}{l|l}{ Ind } & \multicolumn{1}{l|}{ Neg } & \multicolumn{1}{l|}{ Pos } & \multicolumn{1}{l|}{ Total } & \multicolumn{1}{l|}{$\%$} \\
\hline femme & 51 & 10 & 84 & 145 & $13 \%$ \\
\hline homme & 204 & 44 & 685 & 933 & $87 \%$ \\
\hline Total & 255 & 54 & 769 & 1078 & $100 \%$ \\
\hline$\%$ & $24 \%$ & $5 \%$ & $71 \%$ & $100 \%$ & \\
\cline { 1 - 5 } & & &
\end{tabular}

Tableau 3 : La polarité des segments axiologiques selon la tête du syntagme (fréquences et pourcentages)

Au sein de nos observables, la polarité négative peut être associée, avec une assurance variable, à environ $5 \%$ des énoncés. Ce très faible nombre d'attestations à polarité négative constitue un résultat notable qu'il convient d'expliquer. Numériquement peu important (40 termes) et assez uniformément distribué, le lexique qui sature la construction ne révèle pas de tendances fortes. Quelques régularités sémantiques peuvent toutefois être esquissées. On y atteste des substantifs qui dénotent dans l'expression complète des états affectifs (peine, détresse, douleur, nerfs), des formes de manipulation (manouvres, intrigues, coups politiques, mauvais coups, pirouettes...), des jugements moraux (mauvaise vie, orgueil, petite vertu, peu de foi...), des états de faiblesse (faille, contradictions, paradoxes, paille ...).

(11) Et de ce monstre, B., comme Berg, parvient miraculeusement à faire l'être humain par excellence, homme de douleur, qui mérite compassion, respect et presque admiration.

(12) Homme d'orgueil, né sur une terre où la violence s'alimente à des racines millénaires, vivant encore mentalement dans un passé où les pratiques qu'on lui reproche légitimement étaient pas seulement en Orient monnaie courante, S. tient à l'évidence pour fariboles hypocrites le vertueux langage de ses adversaires sur la loi internationale et les droits de l'homme.

Dans la catégorie (Ind, pour 'Indéterminée a priori'), nous avons classé les expressions dont la charge axiologique apparaît comme instable, c'est-à-dire comme nettement dépendante du contexte (13-14). Il est clair que les frontières de cette catégorie sont placées subjectivement. Nous y avons inclus les termes tels que influence, foi, pouvoir, réseau, coulisses, poigne, combat, fer, piété... (les quatre premiers représentent un tiers des attestations); au total nous en avons relevé une centaine.

(13) Qualifié de "pizza froide » par la presse américaine, K.O., soixante et un ans, n'était pas l'homme que les Japonais, déprimés par la crise, attendaient. Jugé terne et triste, homme de 
coulisses, sans compétence économique, le ministre des affaires étrangères d'Hashimoto était le moins populaire de trois candidats à la succession du premier ministre, après l'échec du Parti libéral démocrate (PLD, au pouvoir depuis quarante ans) aux élections partielles de juillet 1998. Conscient de ses faiblesses, M. Obuchi s'est qualifié lui-même de "boeuf empesé » et de " M. Ordinaire ».

(14) LA dame répudiée... M.T., homme d'Etat, femme de fer.

Enfin, plus de 160 termes différents entrent dans les expressions à polarité positive (15-16). Leurs fréquences sont plus fortement différentiées, pouvant aller jusqu'à 65 attestations (pour conviction). Les 20 premiers sont présents dans $60 \%$ des énoncés, avec en tête conviction, culture, action et dialogue.

(15) Homme de conviction, ne manquant pas de panache, communicateur hors pair, L.B. - qui aura soixante ans dans quelques jours - jouit depuis des années d'un grand charisme personnel dans l'opinion publique québécoise.

(16) Homme de dialogue, le dalä-lama reste, envers et contre tout, fidèle à la non-violence, et se garde de la moindre haine envers les Chinois, qui ont annexé le Tibet.

\subsubsection{Qualités saillantes}

Les segments axiologiques se caractérisent par une relative ${ }^{10}$ richesse des qualités pouvant être associées aux cibles. Nous avons attesté près de 300 substantifs différents. Le tableau 4 en présente vingt, par ordre décroissant de fréquence (présents dans $47 \%$ des attestations).

\begin{tabular}{|l|r|r|r|}
\hline qualité & Femme & \multicolumn{1}{l|}{ Homme } & \multicolumn{1}{l|}{ Occ } \\
\hline conviction & 5 & 60 & 65 \\
\hline culture & 2 & 56 & 58 \\
\hline action & 2 & 47 & 49 \\
\hline dialogue & & 38 & 38 \\
\hline influence & 5 & 25 & 30 \\
\hline ordre & 14 & 26 & 29 \\
\hline caractère & 22 & & 25 \\
\hline tête & & 20 & 22 \\
\hline foi & 3 & 17 & 20 \\
\hline pouvoir & & 17 & 17 \\
\hline cœur & 1 & 16 & 17 \\
\hline communication & 1 & 17 & 17 \\
\hline consensus & 5 & 16 & 17 \\
\hline réseau & 1 & 13 & 16 \\
\hline passion & & 14 & 14 \\
\hline expérience & 3 & 9 & 12 \\
\hline ouverture & 1 & 11 & 12 \\
\hline goût & 2 & 9 & 11 \\
\hline honneur & & & \\
\hline autorité & & & 14 \\
\hline
\end{tabular}

Tableau 4 : Substantifs dénotant des qualités axiologiques (extrait).

Le lexique à teneur axiologique recouvre plusieurs champs sémantiques. Tenant compte des affinités entre les termes et de leur fréquence dans le corps des observables, nous avons identifié trois grands 
ensembles de valeurs. Portées par un faible nombre de termes (donnés ci-dessus), ces qualités sont exprimées dans plus de $60 \%$ des énoncés. Il s'agit :

- des qualités relationnelles (dialogue, consensus, compromis, conciliation, contact, communication, confiance, ouverture, paix, parole, cour...),

- du rapport à l'action: ténacité (conviction, caractère, tête, engagement, devoir, ordre, rigueur, principes, décision...) et capacité (action, expérience, métier, défi, progrès ...),

- $\quad$ du bagage culturel et intellectuel (culture, esprit, goût, idées, réflexion...).

Comme on peut le voir dans les énoncés (17-20), les qualités en question, souvent coordonnées entre elles, entrent aussi en résonance avec d'autres éléments de l'énoncé (adepte de l'efficacité dans 20, par exemple).

(17) Homme de terrain, attentif au plus petit détail, homme de contact et de communication, ouvert aux problèmes de chacun, il mène une vie épuisante que sa réussite à la tête de France Loisirs va rendre plus mouvementée encore.

(18) Homme de conviction et de rigueur, J.E. aimait aussi le divertissement de qualité.

(19) S'il avait renoncé à une grande fresque sur ce sujet pour préférer un récit bref, et parfait, L., homme de culture et d'intense curiosité, n'a pas reculé devant une entreprise immense, une anthologie de La Mer dans la littérature française.

(20) M.V.A., adepte de l'efficacité, homme de décision et d'exécution, est apparu comme la personne idoine.

\subsubsection{Les genres et « leurs » qualités}

La distribution des qualités entre les deux catégories (homme et femme) est très fortement différentiée. Seulement $10 \%$ des qualités sont associées à l'une et à l'autre des catégories. Mais, même dans ce cas, la balance penche toujours dans un sens ou dans l'autre, comme on a pu le voir dans le tableau 4.

Les qualités le plus souvent associées aux femmes sont exprimées par les termes tête, caractère, aujourd'hui, conviction, influence, passion, intérieur et poigne ( $46 \%$ des attestations les concernant). On peut y voir la prédominance de la ténacité (21), au sens de (Martin et White, 2005).

(21) Madame le maire, M.-R.T. (PS), contestée sur sa gauche comme sur sa droite, reste sourde aux exigences qui viennent de partout et qui la pressent de démissionner. Mais, femme de caractère, têtue et fière de l'être, elle s'accroche et refuse.

Si l'on choisit, de manière analogue, de ne considérer que les huit premières qualités attribuées aux hommes, elles s'expriment au moyen des substantifs conviction, culture, action, dialogue, ordre, influence, foi et cœur que l'on trouve (dans 31\% des attestations). Plus généralement, en tenant compte de l'ensemble des attestations, on note la prégnance des qualités relationnelles (22) et l'importance du bagage culturel.

(22) Homme de débat, enthousiaste et chaleureux, cette grande figure de la jeune génération qui participa à la démocratisation du Japon après la défaite était restée un homme de son temps, vivant et commentant ses mutations.

Cette asymétrie manifeste dans l'attribution des qualités demande à être discutée dans un cadre plus large, avec les outils de la sociolinguistique et de l'anthropologie. Signalons l'existence des recherches dans le champ des «gender studies » s'attachant notamment à mettre au jour des stéréotypes de la différence sexuelle dans le langage. (Bailly 2008), par exemple, montre comment ces stéréotypes persistants influencent non seulement la façon dont nous parlons et ce que nous disons, mais aussi la façon dont nous percevons et interprétons les paroles d'autrui. 
Pourquoi atteste-t-on femme de tête et homme de cour et non l'inverse ? Remarquons que le dictionnaire du CNRTL ne connaît que les expressions femme de tête et femme de cœur qu'il oppose entre elles. ${ }^{11}$ Cette question, nous ne pouvons que la formuler, en la tenant pour fondée à la lumière des résultats quantitatifs obtenus. Les travaux de (Baider et Jacquey 2008) fournissent des pistes pour une telle recherche, en mettant en avant les différences de significations associées aux mots femme et homme, explicitées sous forme de QUALIAS du Lexique Génératif de Pustejovsky (1995).

\subsection{Segments qualifiants (QI)}

Cette catégorie occupe à l'évidence une place intermédiaire entre celle qui réunit les qualités (ou propriétés) subjectives à caractère axiologique et celle qui rassemble les qualités plus objectives. Et, il existe certainement un continuum au sein même de cette classe. Les expressions telles que homme de dossiers, homme de terrain paraissent plus subjectives (ou moins catégorisantes) que par exemple homme de presse ou homme de sciences (23 vs 24 ).

(23) Homme de dossiers, perspicace et tolérant dont le seul défaut est de ne jamais s'être mis en valeur, il va devoir, désormais, à cinquante-six ans, faire ses preuves dans un poste d'autorité.

(24) Romancier, cinéaste, homme de radio, de télévision... P.L. a construit son destin sur cet éclectisme.

Dans 828 attestations, on relève environ 90 termes différents pouvant qualifier homme ou femme. Statistiquement, la variabilité lexicale est nettement moins importante que celle constatée dans les segments axiologiques, et la fréquence moyenne des termes bien plus élevée $(9,2$ contre 3,6$)$. Le tableau 5 présente vingt termes les plus fréquents. Attestés dans 713 énoncés, ils représentent $86 \%$ de toute la catégorie. A l'intérieur de cette sélection, comme dans toute la catégorie (Q1), les attestations qualifiant les femmes représentent seulement $6 \%$ des énoncés.

\begin{tabular}{|l|r|r|r|}
\hline qualité & femme & homme & \multicolumn{1}{l|}{ Occ } \\
\hline théâtre & 1 & 129 & 130 \\
\hline lettres & 28 & 84 & 112 \\
\hline gauche & 5 & 89 & 94 \\
\hline terrain & 5 & 73 & 78 \\
\hline radio & 1 & 54 & 55 \\
\hline appareil & 1 & 29 & 30 \\
\hline droite & 2 & 28 & 30 \\
\hline presse & & 27 & 27 \\
\hline télévision & & 25 & 25 \\
\hline scène & 2 & 17 & 19 \\
\hline dossiers & & 18 & 18 \\
\hline spectacle & & 17 & 17 \\
\hline science & 1 & 15 & 16 \\
\hline plume & 2 & 13 & 15 \\
\hline guerre & & 9 & 9 \\
\hline médias & & 9 & 9 \\
\hline cinéma & & 8 & 8 \\
\hline entreprise & & 8 & 8 \\
\hline jazz & & 6 & 7 \\
\hline images & & 65 & 713 \\
\hline
\end{tabular}

Tableau 5 : Les principaux attributs qualifiants (extrait) 
Sur le plan sémantique, les substantifs attestés renvoient à plusieurs univers d'activités, liés pour l'essentiel à la culture et à la politique ${ }^{12}$. Y sont représentés notamment :

- l'univers des médias (presse, radio, télévision, médias, programme, studio, plateau...),

- le monde des arts et du spectacle (lettres, plume, scène, spectacle, cinéma, images, jazz, théâtre...),

- le champ de la politique (gauche, droite, gouvernement, parti, ministère, cabinet, administration, appareil ...),

- dans une moindre mesure, l'univers du travail et de l'entreprise (terrain, dossier, entreprise, marketing, marché...).

Quand la construction «(homme|femme) de $\mathrm{N}$ » correspond à un prédicat qualifiant, la signification associée présente la cible comme familière ou experte de l'univers désigné par $\mathrm{N}$. Est dit homme de théâtre quelqu'un qui connaît (très bien / parfaitement...) le monde du théâtre. A l'évidence, cette connaissance experte, selon la perception sociale du domaine concerné, peut être considérée comme une valeur.

\subsection{Segments catégorisants (Ob)}

Cette dernière catégorie renvoie aux emplois qui ne peuvent être classés comme axiologiques ou qualifiants, et de ce fait ne sont pas pertinents pour notre étude. Néanmoins, numériquement significatifs dans le corpus (29\% des attestations), il est important de les caractériser, pour pouvoir les écarter des collectes automatiques.

Deux cas de figure sont constatés. Les expressions « homme|femme de $\mathrm{N}$ » renvoient à des métiers ou des fonctions pouvant être exercés par la personne cible (affaires, ménage, service, chambre, cantine, café) (ex. 25). On est alors en présence d'un petit nombre de formes lexicalisées, qui représentent à elles seules 765 attestations $(90 \%$ de la catégorie). Les statistiques détaillées montrent une répartition clairement sexuée de ces occupations homme d'affaires (620 attestations), femme d'affaires (34), femme de ménage (111), homme de ménage (11), femme de chambre (16), homme de chambre (0).

(25) C'est l'histoire de Madame L., femme de ménage, qui s'affiche en tailleurs pimpants, achetés avec ses gains boursiers... au Printemps, de Shanghaï.

La variante « femme de $\mathrm{N}$ » peut signifier également « épouse/compagne de $\mathrm{N}$ » (64 attestations, soit 8\% de la catégorie $\mathrm{Ob}$ ), avec pour substantifs des termes désignant le métier ou la fonction du compagnon : gendarme, ministre, banquier, président, peintre, ouvrier, marin, agriculteur, flic, boss, rabbin, immigré, harki, colon, prisonnier, détenu, voyou... Une trentaine de termes ont été prélevés sur le corpus. Ce tour sert à caractériser une femme par la profession ou la condition de son mari. En tant qu'indicateur de la position sociale, cette caractéristique semble faire (encore) partie des attributs saillants attachés au genre féminin. Nous sommes ainsi en présence d'un jugement stéréotypé (26).

(26) HANO"I 1920, deux Vietnam, deux vies et deux femmes qui se sont croisées mais que tout séparait. L'une, Française, femme de colon, chapeautée, gantée et habillée de blanc. L'autre, Vietnamienne, paysanne rebelle du delta du fleuve Rouge, vêtue d'un pantalon noir et d'une tunique.

Enfin, dans les $2 \%$ des attestations qui restent, on trouve quelques expressions qui renvoient aux caractéristiques physiques de la personne (couleur, petite taille, solide corpulence...).

Il est à noter que du fait du caractère restreint du lexique concerné (ce qui est logiquement corrélé avec une forte fréquence de certaines formes) ces emplois peuvent assez facilement être identifiés par des procédures automatisées et différenciés des expressions potentiellement axiologiques. 


\section{5 « (Homme|femme) de N » en cotexte}

En complément des analyses sémantiques qui portent sur le composant «qualifiant» $(\mathrm{N})$, nous allons nous intéresser maintenant au cotexte qui environne l'expression complète. Nous donnerons des statistiques sur la position du syntagme au sein de l'énoncé, ainsi que sur son voisinage au sein de l'apposition.

\subsection{Position dans l'énoncé}

La structure «(hommeffemme) de $\mathrm{N}$ » en apposition détachée peut figurer à l'initiale de l'énoncé, en position médiane ou en position finale.

\begin{tabular}{|l|r|r|}
\hline Position & \multicolumn{1}{|l|}{ Total } & \multicolumn{1}{l|}{$\%$} \\
\hline FIN & 396 & $14 \%$ \\
\hline INC & 1481 & $51 \%$ \\
\hline INI & 1010 & $35 \%$ \\
\hline Total & 2887 & $100 \%$ \\
\hline
\end{tabular}

Tableau 6: Les fréquences des expressions P1, selon les positions initiale, médiane ou finale.

Comme on le voit dans le tableau 6, plus d'un tiers des attestations sont placées à l'initiale absolue. La frontalité du segment présuppose une forte accessibilité référentielle de la cible, venant du cotexte gauche (phrases qui précèdent, titre...). La position médiane $(51 \%)$, correspond en réalité à plusieurs configurations différentes : (i) l'insertion entre le sujet et le verbe (27), (ii) le détachement à gauche du sujet, au sein d'une apposition composée de plusieurs segments (28), (iii) dans un énoncé de structure complexe, où l'un des actants (sujet ou objet) est cible de qualifications multiples. Placée en finale de l'énoncé (14\% des cas), l'expression peut qualifier l'actant objet (29), mais aussi l'actant sujet (30).

(27) Gaston III de Foix, dit Gaston Fébus (ou Phoebus), homme de guerre, batailla pour la souveraineté de sa principauté, statut qui ne changera qu'avec l'annexion à la couronne de France par Louis XIII, en 1620. Homme de lettres, il écrivit un Livre des oraisons ainsi qu'un Livre de la chasse, dont il subsiste une quarantaine de versions.

(28) Fidèle à son tempérament chaleureux, homme de contact, P.V. sillonne alors la France entière et fourmille de projets pour réveiller le militantisme $d u P R$.

(29) Mme O. a cédé son restaurant à son maître d'hôtel, M.M., homme de métier

(30) M.A. est femme, femme de tête.

\subsection{Rapport au voisinage}

Comme en témoignent de nombreuses attestations livrées tout au long de cette étude, les constructions « (homme|femme) de $\mathrm{N}$ » peuvent entrer dans des structures apposées complexes (31), pouvant condenser des qualifications et des appréciations.

(31) Homme de concept, pédagogue infatigable, militant sincère, sans l'ombre d'une complaisance pour l'argent, $R$. prolonge à bien des égards la méthode qui séduisait tant chez Pierre MendèsFrance : le langage direct, la passion d'expliquer, le refus des compromissions et des politiques à la petite semaine.

Nous avons cherché à vérifier cette intuition sur un sous-ensemble d'énoncés prélevés du corpus des observables. Un échantillon a été constitué en choisissant parmi les énoncés polarisés, 50 premiers énoncés en homme et 50 premiers énoncés en femme. Tous ces énoncés contiennent donc au moins un segment axiologique. Comme on peut le lire dans le tableau 7, 50\% des énoncés contiennent au moins deux segments détachés. Et on peut en attester jusqu'à 7 . 


\begin{tabular}{|c|c|}
\hline NbrConst & Occ \\
\hline 1 & 50 \\
\hline 2 & 28 \\
\hline 3 & 11 \\
\hline 4 & 6 \\
\hline 5 & 2 \\
\hline 6 & 1 \\
\hline 7 & 2 \\
\hline Total & 100 \\
\hline
\end{tabular}

Tableau 7: Les fréquences des attestations, sur un échantillon, de «homme|femme de $\mathrm{N}$ » axiologique selon le nombre de segments détachés évaluatifs présents dans le même énoncé.

En moyenne, on décompte 1,9 constituants par énoncé. Cela veut dire qu'approximativement, à chaque segment axiologique de forme "homme|femme de $\mathrm{N}$ » est associé au sein du même énoncé un autre segment détaché. On constate notamment que les constructions avec des constituants détachés multiples marient aisément des segments évaluatifs et des segments catégorisants (32).

(32) Mais X.F., femme de tête, dramaturge et auteur du livret, a voulu inverser la vapeur en retrouvant le cours originel de cette très ancienne légende chinoise, dont on trouve les traces dans un roman anonyme du temps des Ming.

A la lumière de ces observations, on retiendra que la construction "homme|femme de $\mathrm{N}$ », simple dans sa forme et relativement facile à reconnaître automatiquement, constitue un indice précieux pour identifier des énoncés ou séquences d'énoncés riches en information évaluative (33).

(33) Car celle-ci, femme de caractère, perçue de façon générale comme intelligente, à cheval sur ses compétences, n'en est pas à son coup d'essai.

Enfin, à l'échelle du texte, des jeux de parallélismes reposant sur l'identité de structure permettent de mettre en évidence des oppositions et des complémentarités entre des qualités d'une même personne (1, 27, 34) ou celles qui sont attribuées à des personnes différentes (2).

(34) La femme qui a le plus fasciné les Américains l'an dernier, C.B.K., a commencé sa carrière chez Calvin Klein. Femme-femme longiligne à la beauté sans artifices, elle a épousé John Kennedy, le célibataire le plus convoité d'Amérique, sur une île quasi déserte lors d'une cérémonie simplissime dont n'a filtré qu'une seule et unique photo, répétée à l'envi dans les magazines du monde entier. Femme de tête, on lui prête une forte personnalité et une bonne pointe de sens de l'humour. Femme de pouvoir, elle a rapidement gravi les échelons chez Calvin Klein. D'abord vendeuse dans la boutique du couturier à Boston, puis chargée des clients célèbres à New York jusqu'à faire partie du petit cercle de Calvin Klein, sa deuxième épouse Kelly et sa fille Marcie jouant même, dit-on, un rôle de muse. Femme-mystère, sa capacité à éluder les médias tout en sachant y briller lorsqu'ils sont inévitables, suscite un parallèle auquel peu de chroniqueurs ont résisté : c'était là l'un des grands talents de celle qui aurait été sa belle-mère si elle n'avait succombé à un cancer en 1994, Jackie Kennedy-Onassis.

Dans tous les cas, il s'agit d'un regard analytique qui pose son objet et en énonce, de manière synthétique, les caractéristiques jugées saillantes et/ou valorisantes.

\section{Pour conclure}

La construction «(homme|femme) de $\mathrm{N}$ » en position détachée est principalement dédiée à l'acte de qualification, lequel dans près de $40 \%$ des cas se charge d'une axiologie positive ou négative. Elle s'insère souvent dans des structures appositives multiples, entièrement évaluatives ou panachant 
évaluation et qualification. L'axiologie positive est largement dominante. Les valeurs axiologiques les plus fréquentes touchent à la qualité des rapports sociaux, de l'expérience professionnelle et de l'implication dans l'action. Les emplois qualifiants, axiologiquement neutres, se caractérisent par une plus grande spécialisation sémantique, avec une focalisation sur l'appartenance experte à des univers artistiques ou politiques. Dans les deux cas, la construction « homme de $\mathrm{N}$ » est nettement plus fréquente que son analogue « femme de $\mathrm{N}$ ». En somme et en simplifiant, dans le corpus analysé, cette expression sert à valoriser des individus de sexe masculin pour leurs capacités relationnelles et leur connaissance investie du monde des arts ou de la politique.

La structure en question appartient de toute évidence à une palette d'éléments langagiers préformés ou «préfabriqués », dans laquelle les auteurs (ici les journalistes) puisent pour exprimer des jugements évaluatifs. On remarque que les lexiques effectivement employés pour la saturer sont relativement restreints. Nous avons noté quelques associations privilégiées entre unités lexicales. Des tours tels que homme de confiance, homme de conviction, homme de terrain, homme de gauche se détachent par leur fréquence au sein de notre corpus, mais on peut s'attendre à les retrouver dans des proportions comparables à une échelle plus vaste ${ }^{13}$.

Sur le plan linguistique, plusieurs études sont possibles, voire nécessaires, à partir de ce premier travail exploratoire et descriptif. Tout d'abord, il serait éclairant et utile de le situer dans l'optique des recherches sur les termes binominaux, notamment dans le cadre des grammaires de construction (Traugott 2008). La classification sémantique des substantifs associés aux termes homme et femme peut et doit être affinée, notamment en faisant appel aux catégories de (Flaux et Van de Velde 2000). L'usage systématique de tests linguistiques (paraphrases avec le verbe avoir, par le génitif de qualité, par des expressions locatives...) permettra de construire des classifications sémantiques plus élaborées et plus solides. Une étude approfondie des rapports qu'entretiennent ces segments avec leur co-texte, en poursuivant ce qui a été amorcée sur les relations sémantiques dans (Jackiewicz 2010), est également à prévoir.

D'un point de vue du TAL, l'implémentation des patrons correspondant aux constituants détachés à caractère axiologique, dont «homme|femme de $\mathrm{N}$ », avec la plate-forme SémioLabs de la société Noopsis, est en cours de réalisation dans le cadre du projet OntOpiTex. Ce travail permettra aussi de se confronter à d'autres corpus, liés à d'autres pratiques socio-culturelles et professionnelles, visibles notamment via le Web.

\section{Références bibliographiques}

Amossy, R., Herschberg Pierrot, A. (2011). Stéréotypes et clichés. Langue, discours, société. Paris : Armand Colin.

Baider, F., Jacquey E. (2008). Modélisation sémantique, sens différentiel et genre, CMLF2008, 1919-1930.

Bailly, S. (2008). Les hommes, les femmes et la communication: mais que vient faire le sexe dans la langue? Paris : Editions L'Harmattan.

Bednarek, M. (2009). Language pattern and ATTITUDE. Functions of Language, 16, 2, 165-192.

Boszormenyi-Nagy, I., Spark, G.M. (1973). Invisible loyalties: Reciprocity in intergenerational family therapy. New York : Harper \& Row.

Bourdieu, P. (1972). Esquisse d'une théorie de la pratique, Genève : Droz.

Charaudeau, P. (1992). La grammaire du sens et de l'expression. Paris : Hachette Education.

Charaudeau, P. (2011). Les médias et l'information. L'impossible transparence du discours. Bruxelles : De Boeck Ina coll.

Emler, N. (1994). La réputation sociale. In Moscovici S. (éd), Psychologie sociale des relations à autrui, Paris : Nathan, 119-139.

Ferrari, S., Charnois, T., Jackiewicz, A., Gardin, P., Widlöcher, A. (2009). Discours évaluatif : une campagne d'annotation pour la validation de patrons. Actes des 6 e Journées Internationales de Linguistique de Corpus JLC, Lorient, France. 
Flaux, N., Van de Velde, D. (2000). Les noms en français : esquisse de classement. Paris : Ophrys.

Jackiewicz, A. (2010). Structures avec constituants détachés et jugements d'évaluation. Document Numérique, 13, 3, $11-40$.

Jackiewicz, A., Charnois, T., Ferrari, S. (2009). Jugements d'évaluation et constituants périphériques. Actes de TALN09, 16e conférence sur le traitement automatique des langues naturelles, Senlis, France.

Kerbrat-Orecchioni, C. (2002). L'énonciation. Paris : Armand Colin.

Martin, J.R., White, P.R.R. (2005). The Language of Evaluation, Appraisal in English. London \& New York: Palgrave Macmillan.

Le Monde (2002). Le style du Monde. Guide rédactionnel.

Neveu, F. (1998). Études sur l'apposition. Paris : Honoré Champion.

Pustejovsky, J. (1995). The Generative Lexicon. Cambridge (MA) / London (UK) : The MIT Press.

Traugott, E.C. (2008). The grammaticalization of NP of NP constructions. In Bergs, A. \& G. Diewald (eds), Constructions and Language Change, Berlin: Mouton de Gruyter, 23-45.

\begin{abstract}
${ }^{1}$ Le projet ANR (ContInt, 2009 - 2012 ; COORD 016) est intitulé « OntOpiTex : Modèles linguistiques et ontologies. Extraction informatique et caractérisation d'opinions et de jugements d'évaluation dans les textes ». Il réunit les laboratoires: LaLIC/STIH (porteur du projet), GREYC et le CRISCO, ainsi que les sociétés TKM (Grenoble) et Noopsis (Caen). L'objectif principal d'OntOpiTex est de chercher à identifier et agréger des segments textuels porteurs d'opinions, et surtout de les classer par type (affect, jugement, appréciation...) et de les caractériser par des critères plus fins : polarité, intensité, prototypicalité, cible(s), source(s), engagement de l'auteur, etc. Des outils interactifs sont proposés pour visualiser et naviguer dans ces annotations à l'échelle d'une collection de documents.
\end{abstract}

${ }^{2}$ Le concept de loyauté a été introduit dans le champ des psychothérapies familiales par Ivan Boszormenyi-Nagy, psychothérapeute et fondateur de la thérapie contextuelle, croisement entre l'approche systémique et la psychanalyse. Le concept de loyauté sert à décrire le lien résistant et profond unissant entre eux les membres d'une même famille, lien qui transcende tous leurs conflits. La loyauté est vue comme une force régulatrice des systèmes. (BoszormenyiNagy et al., 1973).

${ }^{3}$ « [...] l'habitus est le produit du travail d'inculcation et d'appropriation nécessaire pour que ces produits de l'histoire collective que sont les structures objectives (e. g. de la langue, de l'économie, etc.) parviennent à se reproduire, sous la forme de dispositions durables, dans tous les organismes (que l'on peut, si l'on veut, appeler individus) durablement soumis aux mêmes conditionnements, donc placés dans les mêmes conditions matérielles d'existences. » (Bourdieu, 1972, 282)

${ }^{4}$ Le symbole (+exp) renvoie à une expansion éventuelle du terme $\mathrm{N}$ (femme d'exception vs femme d'exception à esprit incandescent)

${ }^{5}$ Dans la suite du document, nous utiliserons la notation simplifiée « homme|femme de $\mathrm{N} »$.

${ }^{6}$ L'ensemble des exemples donnés dans l'article proviennent de ce corpus.

${ }^{7}$ Il s'agit bien d'une propension et non d'une règle stricte. (Cf. Frantext : L536/ Coppée.F / La bonne souffrance /1898, Page 91 : ..., femme de virile intelligence et de jugement haut et sûr, elle...)

${ }^{8}$ Pour (Emler 1994, 125-126), la réputation (i) «porte sur des qualités humaines variables », (ii) « repose sur des qualités individuelles importantes pour l'ensemble de la collectivité », (iii) vise le plus souvent «les qualités dont l'évaluation la plus efficace nécessite un large échantillon de performances réalisées dans différentes circonstances, pendant un certain temps », (iv) « s'établit sur la base des qualités rares, de qualités exceptionnelles ».

${ }^{9}$ Sur la question de la visibilité des femmes dans l'espace médiatique, on peut consulter le rapport «Image des femmes dans les médias », rédigé par Brigitte Grésy, disponible sur http://www.ladocumentationfrancaise.fr/var/storage/rapports-publics//084000614/0000.pdf, consulté le 15 mars 2012.

${ }^{10} \mathrm{Au}$ regard des deux autres catégories $(\mathrm{Q} 1)$ et $(\mathrm{Ob})$. 
${ }^{11}$ http://www.cnrtl.fr/definition/femme: «-Femme de tête. Femme que caractérise une intelligence servie par une volonté ferme, à quoi s'ajoute souvent un réalisme aigu et un sens développé de ses intérêts (p. oppos. à femme de cœur, infra 3).»; "-Femme de cœur. Femme dotée de grandes qualités de cœur (p. oppos. à femme de tête, supra) »; la page http://www.cnrtl.fr/definition/homme ne mentionne pas les expressions homme de tête et homme de cour.

${ }^{12}$ Cette tendance a à voir avec la nature du corpus (le Monde).

${ }^{13} \mathrm{Cf}$. les résultats issus de quelques recherches simples, réalisées sur Internet via Google, le 2 août 2010 : homme de confiance (environ 1090000 résultats), homme de terrain (741 000), homme de gauche (256 000), homme de métier (233 000), homme de conviction(s) (168 000+48 600), homme de tête (114000), homme de droite (107 000) comparés à femme de confiance (285 000), femme de terrain (234 000), femme de tête (124 000), femme de gauche (31 400), femme de conviction(s) (25 700+ 7 390), femme de droite (15 100), femme de métier (1 230)... 